\title{
ALMOST PERIODICITY IN SEMIFLOWS
}

\author{
GEORGE SEIFERT
}

(Communicated by Hal L. Smith)

\begin{abstract}
For a semiflow on a complete metric space we show that if a semiorbit has compact closure and its positive limit set consists of stable points, the orbit is asymptotically almost periodic. Under stronger hypotheses which are more tractable in applications, the same conclusion holds. An application to a scalar delay-differential equation is given.
\end{abstract}

In this paper we deal with a semiflow on a complete metric space $\{X, d\}$; i.e., with a function $\Phi$ continuous on $R^{+} \times X$ to $X$, where $R^{+}$denotes the set of nonnegative real number. If for $(t, x) \in R^{+} \times X$ we denote the function values by $\Phi_{t}(x)$, we assume $\Phi_{0}(x)=x$, and $\Phi_{t_{1}+t_{2}}(x)=\Phi_{t_{1}}\left(\Phi_{t_{2}}(x)\right)$ for $\left(t_{1}, x\right)$, $\left(t_{2}, x\right) \in R^{+} \times X$.

We define

(i) $0^{+}(x)=\left\{\Phi_{t}(x): t \geq 0\right\}$,

(ii) $S=\{x \in X$ : for each $\varepsilon>0$ there exists a $\delta(x)>0$ such that $d(x, y)$ $<\delta(x)$ implies $d\left(\Phi_{t}(x), \Phi_{t}(y)\right)<\varepsilon$ for $\left.t \geq 0\right\}$,

(iii) $A P=\{x \in X$ : for each $\varepsilon>0$, there exists an $L=L(\varepsilon, x)>$ 0 such that for each real $a$ there exists a $\tau \in[a, a+L]$ such that $d\left(\boldsymbol{\Phi}_{t+\tau}(x), \boldsymbol{\Phi}_{t}(x)\right)<\varepsilon$ for all $\left.t \geq 0, t+\tau \geq 0\right\}$.

We call $0^{+}(x)$ the semiorbit through $x, S$ the set of stable points, and $A P$ the set of almost periodic points. Definition (ii) is fairly standard (cf. [1], [2]), while (iii) is introduced in [3]. While $S$ could clearly be empty, for semiflows with certain monotonicity properties, $S$ is not only nonempty, but has an interior dense in $X$ (cf. again [1], [2]). The set $A P$ clearly contains the set of periodic points in $X$; i.e., the set of $x$ for which there exists a $\tau>0$ such that $\Phi_{\tau}(x)=x$. In fact, the set $A P$ was introduced in [3] in proposing a more general definition of chaos, in which the condition on periodic points is replaced by a corresponding condition on $A P$ points.

In our main results we require the following additional definition:

(iv) $A A P=\left\{x \in X\right.$ : there exists a $y \in A P$ such that $d\left(\Phi_{t}(x), \Phi_{t}(y)\right) \rightarrow$ 0 as $t \rightarrow \infty\}$.

We call $A A P$ the set of asymptotically almost periodic points, and say that for $x \in A A P, 0^{+}(x)$ is asymptotically almost periodic.

Received by the editors January 15, 1994.

1991 Mathematics Subject Classification. Primary 58F25, 34K15.

(C)1995 American Mathematical Society 
It follows that for a fixed $x \in A A P$, the corresponding $y \in A P$ is unique. To show this we introduce the following notation: for each $\varepsilon>0$ and $x \in X$ we define

(v) $E(\varepsilon, x)=\left\{\tau \in R: d\left(\Phi_{t+\tau}(x), \Phi_{t}(x)\right)<\varepsilon\right.$ for $\left.t \geq 0, t+\tau \geq 0\right\}$; here $R$ denotes the set of real numbers.

From the definition of $x \in A P$, it follows that $x \in A P$ if and only if there exists a $L=L(\varepsilon, x)>0$ such that any real interval of length $L$ contains a point of $E(\varepsilon, x)$; i.e., $E(\varepsilon, x)$ is relatively dense.

Lemma 1. If $x \in A A P$, the corresponding $y \in A P$ is unique.

We outline a proof. It can be established that if $y_{1}$ and $y_{2}$ are $A P$ points, then for each $\varepsilon>0, E\left(\varepsilon, y_{1}\right) \cap E\left(\varepsilon, y_{2}\right)$ is nonempty; this is nontrivial, but can be proved along the lines of a similar result proved in [4]. From this it follows that given $\varepsilon>0$, there exists arbitrarily large $\tau \in R$ such that for $y_{1}, y_{2} \in A P$

$$
d\left(y_{1}, \Phi_{\tau}\left(y_{1}\right)\right)<\varepsilon, \quad d\left(y_{2}, \Phi_{\tau}\left(y_{2}\right)\right)<\varepsilon .
$$

If $d\left(\Phi_{t}(x), \Phi_{t}\left(y_{i}\right)\right) \rightarrow 0$ as $t \rightarrow \infty, i=1,2$, there clearly exists $\tau$ such that (1) holds and also $d\left(\boldsymbol{\Phi}_{\tau}\left(y_{1}\right), \boldsymbol{\Phi}_{\tau}\left(y_{2}\right)\right)<\varepsilon$. So $d\left(y_{1}, y_{2}\right) \leq d\left(y_{1}, \Phi_{\tau}\left(y_{1}\right)\right)+$ $d\left(\Phi_{\tau}\left(y_{1}\right), \Phi_{\tau}\left(y_{2}\right)\right)+d\left(\Phi_{\tau}\left(y_{2}\right), y_{2}\right)<3 \varepsilon$ and, since $\varepsilon$ is arbitrary, $y_{1}=y_{2}$. define

(vi) $\omega(x)=\left\{y \in X: \Phi_{t_{n}}(x) \rightarrow y\right.$ for some sequence $t_{n} \rightarrow \infty$ as $\left.n \rightarrow \infty\right\}$ and refer to $\omega(x)$ as the positive limit set of $x$.

Theorem 1. Let $\overline{0^{+}(x)}$ be compact and $\omega(x) \subset S$. Then $x \in A A P$, and $\omega(x) \subset A P$.

Proof. Let $\left\{t_{n}: n=1,2, \ldots\right\}$ be such that $t_{n} \rightarrow \infty$ as $n \rightarrow \infty$ and $t_{n}>$ $0, n=1,2, \ldots$. Since $\overline{0^{+}(x)}$ is compact, there exists a subsequence $\left\{n_{k}: k=\right.$ $1,2, \ldots\}$ of the sequence of integers $\{n\}$ such that if $\bar{t}_{k}=t_{n_{k}}$, there exists a $y \in \omega(x)$ such that $\Phi_{t_{k}}(x) \rightarrow y$ as $k \rightarrow \infty$. Since $y \in S$, it follows that $\Phi_{t}\left(\Phi_{t_{k}}(x)\right)=\Phi_{t+\bar{t}_{k}}(x) \rightarrow \Phi_{t}(y)$ as $k \rightarrow \infty$ uniformly for $t \geq 0$. By an argument similar to that used in the proof of Theorem 9.3 in [5], we conclude that there exists a function $f: R^{+} \rightarrow X$, uniformly continuous and bounded in $R^{+}$, and such that for each $\varepsilon>0$,

$$
E(f, \varepsilon)=\{\tau \in R: d(f(t+\tau), f(t))<\varepsilon \quad \text { for } t \geq 0, t+\tau \geq 0\}
$$

is relatively dense in $R$ and such that

$$
d\left(\Phi_{t}(x), f(t)\right) \rightarrow 0 \quad \text { as } t \rightarrow \infty .
$$

Let $\varepsilon>0$, and let $\tau_{k} \in E(f, \varepsilon)$ be such that $\tau_{k} \rightarrow \infty$ as $k \rightarrow \infty$; we may suppose by using a suitable subsequence if necessary that $\Phi_{\tau_{k}}(x) \rightarrow \bar{y} \in \omega(x)$ as $k \rightarrow \infty$ and, in fact, also $\Phi_{t+\tau_{k}}(x) \rightarrow \Phi_{t}(\bar{y})$ as $k \rightarrow \infty$ uniformly for $t \geq 0$, since $\bar{y} \in S$. But

$$
\begin{aligned}
d\left(\Phi_{t}(\bar{y}), f(t)\right) \leq & d\left(\Phi_{t}(\bar{y}), \Phi_{t+\tau_{k}}(x)\right) \\
& +d\left(\Phi_{t+\tau_{k}}(x), f\left(t+\tau_{k}\right)\right) \\
& +d\left(f\left(t+\tau_{k}\right), f(t)\right)
\end{aligned}
$$


and so for $k$ sufficiently large

$$
d\left(\Phi_{t}(\bar{y}), f(t)\right)<3 \varepsilon, \quad t \geq 0 .
$$

So $f(t)=\Phi_{t}(\bar{y}), t \geq 0$, and so $\bar{y} \in A P$ by definition, and we have that $x \in A A P$. To show that $\omega(x) \subset A P$, let $z \in \omega(x)$. Then $\Phi_{t_{k}}(x) \rightarrow z$ as $k \rightarrow \infty$ for some sequence $t_{k} \rightarrow \infty$ as $k \rightarrow \infty$. Since $x \in A A P$, there exists $y \in A P$ such that $\Phi_{t}(x)-\Phi_{t}(y) \rightarrow 0$ as $t \rightarrow \infty$. Since $y \in A P$, there exists a sequence $\tau_{k} \rightarrow \infty$ as $k \rightarrow \infty$ such that $\Phi_{t_{k}}(y) \rightarrow y$ as $k \rightarrow \infty$. It follows that $\Phi_{\tau_{k}}(x) \rightarrow y$ as $k \rightarrow \infty$. But $\Phi_{t_{k}}(x)-\Phi_{t_{k}}(y) \rightarrow 0$ as $k \rightarrow \infty$, so clearly $\Phi_{t_{k}}(y) \rightarrow z$ as $k \rightarrow \infty$. Since $z \in S, \Phi_{t+t_{k}}(y) \rightarrow \Phi_{t}(z)$ as $k \rightarrow \infty$ uniformly for $t \geq 0$, and since $y \in A P$, we have, by a straightforward argument which we omit, that $z \in A P$.

The existence of $A P$ points can be established in terms of another type of stability which, although stronger, is in certain applications easier to establish than the type used so far.

Definition 1. Let $B$ be a subset of $X$; we say that the semiflow $\Phi$ is equistable on $B$ if given $\varepsilon>0$, there exists a $\delta=\delta(B)>0$ such that $d(x, y)<\delta, x, y \in$ $B$, implies $d\left(\Phi_{t}(x), \Phi_{t}(y)\right)<\varepsilon, t \geq 0$.

Theorem 2. Let $\overline{0^{+}(x)}$ be compact, and let $\Phi$ be equistable on some open set $B \supset \omega(x)$. Then $x \in A A P$ and $\omega(x) \in A P$.

This is a very simple corollary of Theorem 1 since it is easily seen that if $\Phi$ is equistable on $B \subset X$ and $B$ is open, then $B \subset S$. We note, however, that unless $B$ is open, this does not follow.

We apply Theorem 2 to show that certain delay-differential equations have asymptotically almost periodic solutions. In particular, we consider the scalar equation

$$
x^{\prime}(t)=a x(t-1)-f(x(t), x(t-1)), \quad t>0,
$$

with initial condition $x(s)=\psi(s),-1 \leq s \leq 0$, where $\psi$ is a given real-valued function continuous on $[-1,0]$, the set of which we denote by $C[-1,0], a>0$ is a constant, and $f(x, y)$ is continuously differentiable on $R^{2}=R \times R$.

Theorem 3. Suppose

$$
\begin{gathered}
f(0, y)=0 \quad \text { for all } y \\
\frac{\partial f}{\partial x}(x, y) \geq\left|\frac{\partial f}{\partial y}(x, y)\right|+a \quad \text { for }(x, y) \neq(0,0) .
\end{gathered}
$$

Then all solutions of (3) are asymptotically almost periodic.

Proof. It is well known that the solutions of (3) generate a semiflow on $C[-1,0]$ given by $\Phi_{t}(\psi)=x(t+s, \psi),-1 \leq s \leq 0, t \geq 0$, where $x(t, \psi)$ denotes the unique solution of initial value problem (3). It is also known that if $x(t, \psi)$ is a bounded solution on $t \geq 0$, the set $\left\{\Phi_{t}(\psi): t \geq 0\right\}$ has compact closure in $C[-1,0]$. Here we may clearly take the metric $d$ on $C[-1,0]$ in terms of the supremum norm; i.e., for $\psi_{1}, \psi_{2} \in C[-1,0]$,

$$
d\left(\psi_{1}, \psi_{2}\right)=\sup \left\{\left|\psi_{1}(s)-\psi_{2}(s)\right|:-1 \leq s \leq 0\right\} .
$$


We next show that under conditions (3.1)-(3.2), this semiflow will be equistable on all of $C[-1,0]$; we can then conclude from Theorem 2 that all bounded solutions of (3) are asymptotically almost periodic in the sense that each $\psi \in C[-1,0]$ for which $\left\{\Phi_{t}(\psi): \tau \geq 0\right\}$ is bounded is in $A A P$ for this semiflow.

Let $x\left(t, \psi_{1}\right)$ and $x\left(t, \psi_{2}\right)$ be any two solutions of the initial value problem (3), and define $\xi(t)=x\left(t, \psi_{1}\right)-x\left(t, \psi_{2}\right)$. We use the Liapunov-Razumikhin technique with the Liapunov function $V(\xi)=\xi^{2} / 2$ for the equation satisfied by $\xi(t)$ :

$$
\xi^{\prime}(t)=a \xi(t-1)-\frac{\partial f}{\partial x} \xi(t)-\frac{\partial f}{\partial y} \xi(t-1)
$$

where $\frac{\partial f}{\partial x}$ and $\frac{\partial f}{\partial y}$ are evaluated at the point $\left(x\left(t, \psi_{2}\right)+\theta(t) \xi(t), x\left(t-1, \psi_{2}\right)+\right.$ $\theta(t) \xi(t-1))$; here $\theta(t) \in[0,1]$ : we have clearly used the Mean Value Theorem.

Using (4), we have

$$
\begin{aligned}
d V(\xi(t)) / d t & =a \xi(t) \xi(t-1)-\frac{\partial f}{\partial x} \xi^{2}(t)-\frac{\partial f}{\partial y} \xi(t) \xi(t-1) \\
& \leq a \xi^{2}(t)-\left(\left|\frac{\partial f}{\partial y}\right|+a\right) \xi^{2}(t)+\left|\frac{\partial f}{\partial y}\right| \xi^{2}(t)=0
\end{aligned}
$$

whenever $V(\xi(t+s) \leq V(\xi(t)),-1 \leq s \leq 0$. So by the Liapunov-Razumikhin technique (cf. for example [6]) we conclude that

$$
V(\xi(t)) \leq \sup \left\{\left|\psi_{1}(s)-\psi_{2}(s)\right|:-1 \leq s \leq 0\right\} .
$$

This clearly shows that the semiflow $\Phi$ is equistable on $C[-1,0]$. Since we may choose $\psi_{2}(s)=0,-1 \leq s \leq 0$, and so have $x\left(t, \psi_{2}\right)=0$ for $t \geq 0$, this also shows that $x(t, \psi)$ is bounded on $t \geq 0$ for each $\psi \in C[-1,0]$, and so our proof is complete.

We note that as a consequence of (3.1) and (3.2), the equilibrium point $x=0$ of (3) is stable but not necessarily asymptotically stable. It could however be an attractor for all solutions of (3). On the other hand, the special case of (3) with $f(x, y)=a x$ is a linear autonomous delay equation, and by a standard analysis of the associated characteristic equation, we find that 0 is a simple root of this equation and all other roots have negative real parts. Thus in this case, all solutions are asymptotic to some constant. Since each constant is a solution to this special case, none of these equilibrium solutions is asymptotically stable.

Finally, it may be of interest to point out that for flows on $R \times X$, i.e., dynamical systems on $R$, stability conditions that guarantee that a point be an $A P$ point are known (cf. part 2 in [7]). One such result (due to A. A. Markov) mentioned in [7] is in terms of an equistability condition on the orbit of the point. This condition has been used to establish the existence of almost periodic solutions of autonomous ordinary differential equations.

\section{REFERENCES}

1. H. Smith and H. R. Thieme, Quasiconvergence and stability for strongly order-preserving semiflows, SIAM J. Math. Anal. 21 (1990), 673-392.

2. $\_$Convergence for strongly order-preserving semiflows, SIAM J. Math. Anal. 22 (1991), 1081-1101. 
3. G. Seifert, On chaos in general semiflows, submitted for publication.

4. A. S. Besicovitch, Almost periodic functions, Dover, New York, 1954.

5. A. M. Fink, Almost periodic differential equations, Lecture Notes in Math., vol. 337, Springer, New York, 1974.

6. J. K. Hale, Theory of functional differential equations, Springer, New York, 1972.

7. V. V. Nemyskii and V. V. Stepanov, Qualitative theory of differential equations, Princeton Univ. Press, Princeton, NJ, 1960.

Department of Mathematics, Iowa State University, Ames, Iowa 50011

E-mail address: S1.mth@isumvs.bitnet 